\title{
Adhesion Reliability Enhancement of Silicon/Epoxy/Polyimide Interfaces for Flexible Electronics
}

\author{
Sanwi Kim and Taek-Soo Kim ${ }^{\dagger}$ \\ Department of Mechanical Engineering, KAIST 291 Daehak-ro, Yuseong-gu, Daejeon 305-701, Korea
}

(Received September 4, 2012: Corrected September 19, 2012: Accepted September 28, 2012)

\begin{abstract}
Adhesion and mechanical reliability of silicon/epoxy/polyimide interfaces are critical issues for flexible electronics. Bonds between these interfaces are mainly hydrogen bonds, so their adhesion is weaker than cohesive fracture toughness and vulnerable to moisture. In order to enhance adhesion and suppress moisture-assisted debonding, UV/Ozone treatment and innovative sol-gel derived hybrid layers were applied to silicon/epoxy/polyimide interfaces. The fracture energy and subcritical crack growth rate were measured by using a double cantilever beam (DCB) fracture mechanics test. Results showed that UV/Ozone treatment increased the adhesion, but was not effective for improving reliability against humidity. However, by applying sol-gel derived hybrid layers, adhesion increase as well as suppresion of moistureassisted cracking were achieved.
\end{abstract}

Keywords: Adhesion, reliability, sol-gel, UV-Ozone treatment, flexible electronics

\section{Introduction}

Recently, a tremendous amount of interest has been focused on flexible electronics due to their numerous possible uses in the future. Some of the possible applications are lightweight and flexible displays, printed RFIDs, sensors, and transparent portable devices. ${ }^{1,2)}$ Besides these common examples, flexible electronic systems can be applied to examples that are not possible with current wafer-based technology, such as sensory skins and electronic textiles. ${ }^{3)}$ Among many choices of substrates for flexible electronics, polyimide makes an excellent substrate for microelectronics due to many qualities, such as good heat resistance, chemical stability, and good electrical properties. ${ }^{4)}$

However, high stress caused by large coefficient of thermal expansion (CTE) mismatch and deformation by severe bending and stretching, in turn, results in a critical problem for mechanical reliability in the interfaces. Also, for the flexible electronics, as devices are being mounted on flexible substrates, film deformation will occur and stress will increase. Furthermore, fabrication processes and real life situations are generally harsh environments that will result in environmentally assisted crack growth. Among many environment factors, moisture is one of the most important and practical factor to investigate. It is already widely researched that moisture deteriorates epoxy adhesion and moisture cannot be avoided in-service situations. All of these factors will significantly reduce adhesion and reliability of flexible electronics, which highlights the need for enhancement methods for better adhesion and reliability.

Due to its importance in practical engineering, many efforts have been made toward the understanding of this

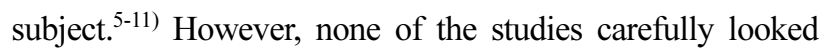
into the fracture energy (critical value of the strain energy release rate) between silicon/epoxy/polyimide interfaces, which is a key data to understand the adhesion and delamination mechanisms. Also, subcritical crack propagation phenomenon in humid conditions is an important factor to investigate. Subcritical crack propagation phenomenon is which a crack propagates slowly under critical value of strain energy release rate due to environmental factors, such as humidity. By investigating these two criteria, it is possible to apply fracture mechanics and understand more about crack propagation and reliability issues concerning humidity for flexible electronics.

The purpose of this paper is to solve problems mentioned above by directly measuring the adhesion between silicon/ epoxy/polyimide interfaces and enhancing adhesion and reliability for flexible electronics in humid condition. The proposed solution in this research is UV/Ozone treatment and innovative sol-gel derived hybrid layers. Beside enhancing adhesion and humidity reliability, which will be proven by this research, UV/Ozone treatment and sol-gel derived hybrid layers provide other benefits, such as being

\footnotetext{
${ }^{\dagger}$ Corresponding author

E-mail: tskim1@kaist.ac.kr
} 
environment friendly and low temperature process adhesive. For example, many surface cleaning processes that include volatile organic solvants were replaced by UV/Ozone treatment due to their harmful and toxic by-products. ${ }^{12)}$ Also, one advantage of sol-gel method is that all the steps involved can be carried out in room temperature. These advantages from UV/Ozone treatment and sol-gel derived hybrid layers provide enormous advantages for the mass production of the flexible electronics. All of these side benefits increase the merit of using UV/Ozone treatment and sol-gel derived hybrid layers for enhancing adhesion and humidity reliability. Beneficial effects of these methods regarding enhancing adhesion was tested by using double cantilever beam (DCB) test to obtain the fracture energy. Also, for enhancing humidity reliability, subcritical crack propagation was investigated by DCB test, which was done under $30^{\circ} \mathrm{C}$ and $85 \%$ humidity condition.

\section{Experimental Procedures}

\subsection{Specimen preparation procedures}

The specimens used in this research had following structure: silicon/GPTMS+TPOZ/ epoxy/GPTMS+APTMS/ PI/GPTMS+APTMS/ epoxy/GPTMS+TPOZ/silicon, which is displayed by Fig. 1 (a). In more detail, the polyimide (PI) substrate was placed in between silicon beams with epoxy being the adhesive, therefore making DCB specimen structure as shown in Fig. 1 (b). In order to enhance the adhesion and humidity reliability of the silicon/epoxy/PI structure, two different sol-gel derived hybrid layers were used for each silicon and PI surfaces. For silicon and epoxy

(a)
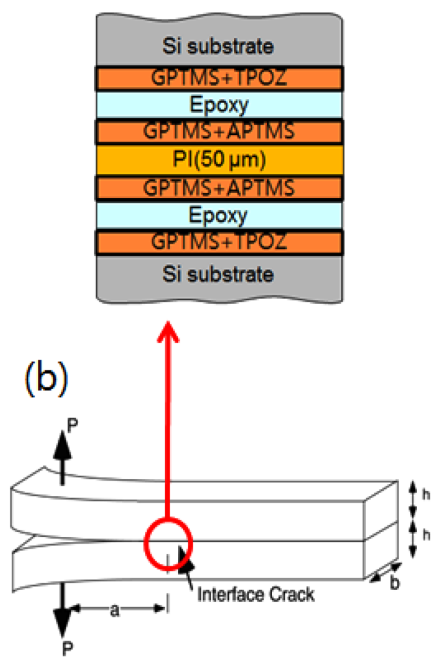

Fig. 1. (a) specimen structure showing interfaces and (b) DCB specimen structure. (a) GPTMS

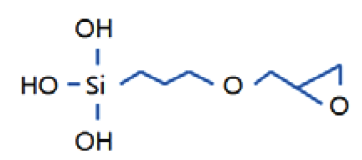

(b) APTMS

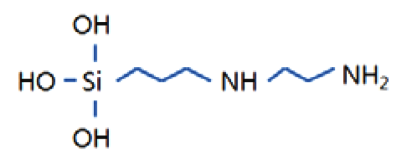

(c)

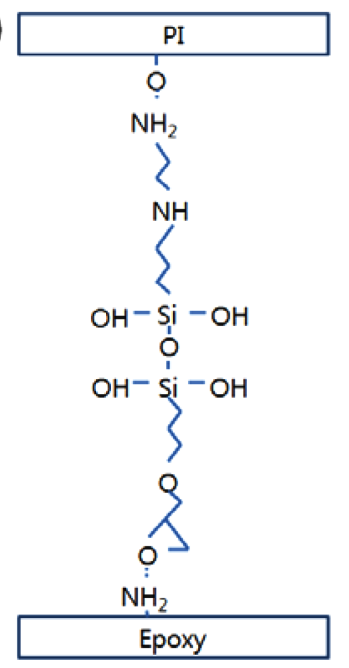

Fig. 2. Chemical structure of (a) GPTMS, (b) APTMS, and (c) combined network of GPTMS and APTMS.

interfaces, sol-gel derived hybrid layer is made by mixing GPTMS (3-Glycidoxypropyltrimethoxysilane) (Gelest, Inc, Morrisville, PA) and TPOZ (Zirconium n-propoxide, 70\% in n-propanol) (Gelest, Inc, Morrisville, PA). This sol-gel derived hybrid layer, which will be named GPTMS+TPOZ for future references, was developed by Oliver et al. for the purpose of increasing the adhesion between silicon and epoxy interfaces. ${ }^{13)}$ For PI and epoxy interfaces, an innovative and novel sol-gel derived hybrid layer was developed to enhance the adhesion and reliability of the target interfaces. This layer consists of GPTMS and APTMS (3-Aminopropyltrimethoxysilane) (Gelest, Inc, Morrisville, PA), which will be referenced as GPTMS+APTMS, and their chemical structures are shown in Fig. 2 (a) and (b). Also, the network structure of sol-gel derived hybrid coupling layer processed from GPTMS and APTMS is shown in Fig. 2 (c). More specific procedures for making above sol-gel derived hybrid layers will be discussed later in a separate sub-section.

This specimen was constructed by following procedures. First, silicon wafers with $<100>$ orientation were cut into small rectangular specimens. The width of the specimen was $10 \mathrm{~mm}$ and length was about $50 \mathrm{~mm}$. PI was also cut 
with the same specimen size. Then, silicon specimen was dip-coated with GPTMS+TPOZ at the velocity of $5 \mathrm{~mm} / \mathrm{s}$ for submerging and $2 \mathrm{~mm} / \mathrm{s}$ for withdrawal. For PI, it was dipcoated with GPTMS+APTMS with $5 \mathrm{~mm} / \mathrm{s}$ for submerging and $2 \mathrm{~mm} / \mathrm{s}$ for withdrawal, as same as GPTMS+TPOZ. Both silicon and PI specimens were then cured for 1 hour at $120^{\circ} \mathrm{C}$. Finally, a selected epoxy (Epo-Tek 353ND consisting of bisphenol $\mathrm{F}$ and imidazole; Epoxy Technology) was applied to two silicon beams. PI substrate was inserted in the middle and the whole structure was given a firm pressure to ensure good adhesion quality. After applying epoxy, the whole specimen was cured for 2 hours at $120^{\circ} \mathrm{C}$.

In this study, the UV/Ozone treatment was introduced as another method for adhesion and reliability enhancement. So, for all experiment specimens, one side of silicon was UV/Ozone treated while both sides were treated for PI before usage. 1 minute was used as both treating conditions and holding time conditions. The reason for using 1 minute as a standard UV/Ozone treating and holding conditions will be mentioned later.

\subsection{Sol-gel derived hybrid layers preparation procedures}

Since there are specific coupling agents for each materials, separate sol-gel derived hybrid layers need to be prepared for each target interfaces. The first sol-gel derived hybrid layer (GPTMS+TPOZ) is known for enhancing the adhesion and the reliability of the interface between silicon and epoxy adhesive. $^{13)}$

The main focus of this paper is the second sol-gel derived hybrid layer (GPTMS+APTMS), which is originally proposed, developed and used in this study. This sol-gel derived hybrid layer's target interface is between PI and epoxy. More detailed formula and procedures are as follows: first, $80 \mathrm{ml}$ of DI water and $0.82 \mathrm{ml}$ of $\mathrm{CH}_{3} \mathrm{COOH}$ is mixed and stirred at $500 \mathrm{rpm}$ by magnetic stirrer. While it is being stirred, $1.180 \mathrm{ml}$ of GPTMS and another $1.180 \mathrm{ml}$ of APTMS are added to the acidic solution, respectively. Once all the chemicals are mixed, the solution is stirred at $500 \mathrm{rpm}$ for 1 hour. After this step, the sol-gel derived hybrid layer's hydrolysis is complete and ready for dip-coating.

\subsection{Double cantilever beam test}

To measure the adhesion strength of the specimen, DCB test was used. The schematics of DCB test specimen is shown in Fig. 1 (b) and the experiment instrument used for DCB test is high-precision micromechanical test system (Delaminator Adhesion Test system; DTS company, Menlo Park, Ca, USA). With this DCB test, quantitative critical value of the strain energy release rate or fracture energy, $G_{c}$, of the specimen can be measured. By knowing this quatitative data, it is possible to use many useful concepts from fracture mechanics to understand more fully about the delamination processes occurring between interfaces of the specimen.

In more detail, DCB test is operated in following manners. First, the specimen is made as Fig. 1 (a) by following the specimen preparation procedures described in subsection 2.1 and 2.2. The tab for applying modeloading by highprecision micro mechanical test system is attached on top of pre-crack area, which is where the black arrow originates in Fig. 1 (b). Once the specimen is inserted into the test system, the displacement between two grips will be increased slowly, usually $1 \mu \mathrm{m} / \mathrm{s}$, and the corresponding load is measured by the load cell located at the opposite side of the actuator. This displacement and load data will be plotted on the graph and this data will be used to obtain crack length, a, and fracture energy, $\mathrm{G}_{\mathrm{c}}$. Crack length and fracture energy will be measured from the slope of the graph by the Eq. (1) and Eq. (2). ${ }^{14,15)}$ In Eq. (1) and Eq. (2), $\mathrm{C}$ is the specimen compliance, $\mathrm{P}_{\mathrm{c}}$ is the critical load where crack-growth starts, $E^{\prime}$ is the plain strain modulus of the silicon, $b$ is the specimen width, and $h$ is the half height of the specimen.

$$
\begin{aligned}
& a=\left(\frac{b C E^{\prime} h^{3}}{8}\right)^{1 / 3}-0.64 h \\
& G_{a}=\frac{12 P_{c}^{2} a^{2}}{b^{2} h^{3} E^{\prime}}\left(1+0.64 \frac{h}{a}\right)^{2}
\end{aligned}
$$

\subsection{Subcritical test}

The fracture energy, $\mathrm{G}_{\mathrm{c}}$, values obtained by the DCB test is the measure of the energy available at the crack extension. Hence, the crack should not propagate until the exceeding amount of energy is applied to the specimen. However, when the specimen is under harsh humidity condition, the crack might extend even when the energy below the fracture energy is applied, which is called the subcritical crack propagation. The subcritical test is designed to measure that exact behavior of the crack, subcritical crack propagation, by using the DCB test settings under certain temperature and humidity conditions. The experiment instrument set-up is as same as the DCB test, except the fact that the DCB test set-up is inserted into the temperature and humidity chamber at $30^{\circ} \mathrm{C}$ and $85 \%$ humidity, which is a more harsh condition for the specimen considering that the DCB test is done at the room temperature and humidity conditions. With this subcritical test set-up, the specimen will be exposed to a stable humidity and temperature environment, thus allowing the measurement of reliable and stable subcritical crack growth rate, da/dt. In this test, the specimen was under 
statically loaded and decreasing $\mathrm{G}$ conditions, which resulted in the range of da/dt from $10^{-5}$ to $10^{-10} \mathrm{~m} / \mathrm{s}$. By using this test, the beneficial effect of sol-gel derived hybrid layers and UV/ Ozone treatment against humidity can be observed.

\section{Results and Discussion}

\subsection{Adhesion enhancement and contact angle measure- ment results}

As discussed above, DCB test was primarily used to determine the adhesion strength of the specimen structure in Fig. 1 (a) and (b). First, the effect of UV/Ozone treatment for adhesion enhancement is investigated and the results are shown in Fig. 3. UV/Ozone holding time was set as 5 minutes for all specimens in this experiment because holding time is a less important factor for adhesion improvement than $\mathrm{UV} /$ Ozone treating time. As the UV/Ozone treating time increased from 0 minute to 20 minutes, the overall trend showed that the fracture energy increased also. Comparing with the fracture energy of no UV/Ozone treating time, equivalent value for 1 minute of $\mathrm{UV} /$ Ozone treating time increased from approximately $87 \mathrm{~J} \mathrm{~m}^{-2}$ to $235 \mathrm{~J} \mathrm{~m}^{-2}$. For 10 minutes of UV/Ozone treating time, the fracture energy increased even more to $290 \mathrm{~J} \mathrm{~m}^{-2}$. Finally, the fracture energy value reached $310 \mathrm{~J} \mathrm{~m}^{-2}$ for 20 minutes of UV/Ozone treating time. Interesting result that can be seen from this graph is that the increase rate of the fracture energy continuously decreased after 1 minute compared with that of 0 minute to 1 minute. This phenomenon is due to the fact that the UV/Ozone treatment's effectiveness decreases as treatment time increases. This result imply that there is an optimum treatment time for increasing the fracture energy.

This optimum UV/Ozone treatment time was investigated by contact angle measurement experiments and the reason for using this particular test is as follows. First of all, the

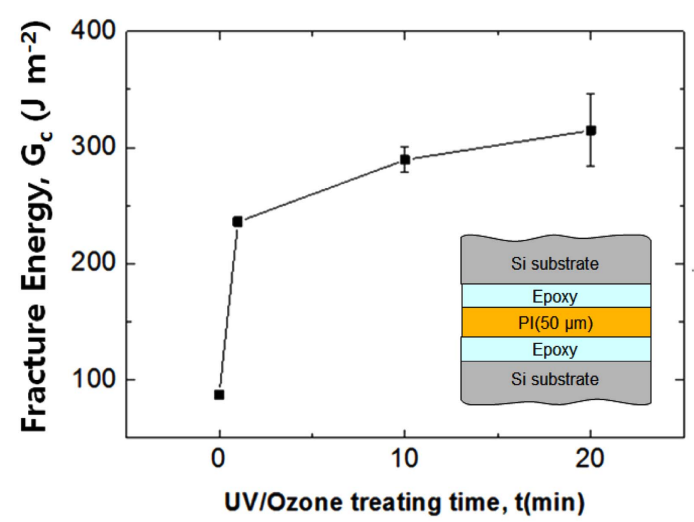

Fig. 3. Measured fracture energy by DCB test for different UV/ Ozone treating time. beneficial effects of UV/Ozone treatment are a) removal of variety of organic contaminants from surfaces, b) increased adhesive strength, c) increased surface wetting, and d) creation of variety of functional groups. ${ }^{16-19)}$ These beneficial effects come in a package, thus checking the surface wetting by contact angle measurements can show how much the overall beneficial effects are introduced to the substrate being investigated. Therefore, contact angle measurements for silicon and PI substrates were done to check the effectiveness of UV/Ozone treatment and their results are shown in Fig. 4 (a) and (b). Fig. 4 (a) is the contact angle of PI for different UV/Ozone treating time and holding time while Fig. 4 (b) is the contact angle of silicon for 0 minute holding time. For silicon, 0 minute holding time is only displayed in Fig. 4 (b) because changing holding time didn't affect contact angle values. Also, the contact angle values of silicon substrate, the angle was already so small, which was about $8 \sim 10 \mathrm{deg}$, that it was hard to measure exactly anymore. Also, for silicon, the UV/Ozone treating time was shown only until 60 seconds because the contact angle values for more than 60 seconds were very similar to that of

(a)

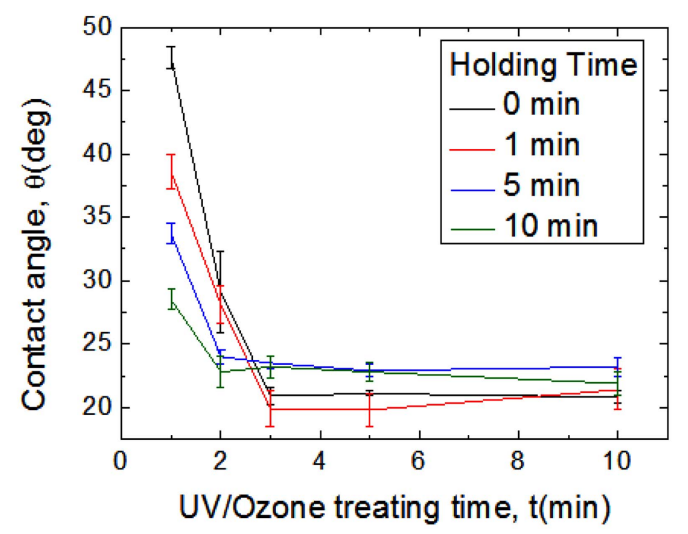

(b)

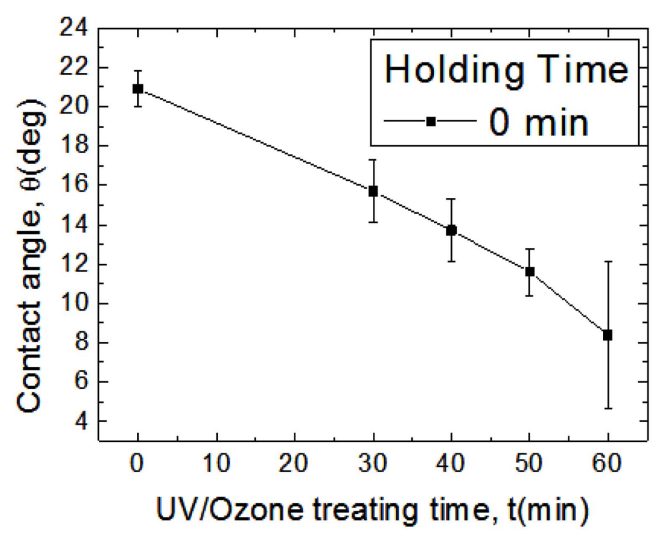

Fig. 4. Contact angle of different UV/Ozone treating time for (a) PI and (b) silicon. 
60 seconds. After 60 seconds, a plateau was found for contact angle values of silicon substrate. Overall, the common trend of Fig. 4 (a) and (b) is that the contact angle decreases very fast only until $1 \sim 3$ minutes. This phenomenon explains why the fracture energy increased greatest between 0 minute and 1 minute of UV/Ozone treating time in Fig. 3. The fracture energy increased greatest between 0 minute and 1 minute in Fig. 3 because UV/Ozone treatment effects were greatest between that time. After 3 minutes, UV/ Ozone treatment had less impact on PI and silicon as proven by Fig. 4 (a) and (b). Hence, the fracture energy increase was not very significant.

Another method of increasing the adhesion further was suggested in this paper as applying sol-gel derived hybrid layers on both silicon and PI surfaces. For silicon surfaces, GPTMS+TPOZ mixture is used while GPTMS+APTMS is used for PI surfaces. GPTMS and APTMS have been chosen as the suitable chemicals since APTMS is a known coupling agent for PI while GPTMS is a coupling agent for epoxy composites. Hence, it was assumed that mixing GPTMS and APTMS will create sol-gel derived hybrid layer networks as seen by Fig. 2 (c) and increase the adhesion.

DCB test was also performed to validate the effect of solgel derived hybrid layers on enhancing the fracture energy. Before applying the sol-gel derived hybrid layers, UV/ Ozone treatment was applied with 1 minute of UV/Ozone treating time and 1 minute of UV/Ozone holding time. The results, which is shown in Fig. 5, shows the overall increase in fracture energy when compared to the fracture energy of specimen treated with only 1 minute of UV/Ozone treatment without any sol-gel derived hybrid layers: from $235 \mathrm{~J} \mathrm{~m}^{-2}$ to average of $304.73 \mathrm{~J} \mathrm{~m}^{-2}$. This result displays the overall adhesion enhancement for the target specimen. From this observation, it can be concluded that using sol-gel derived hybrid layer increased the adhesion even further.

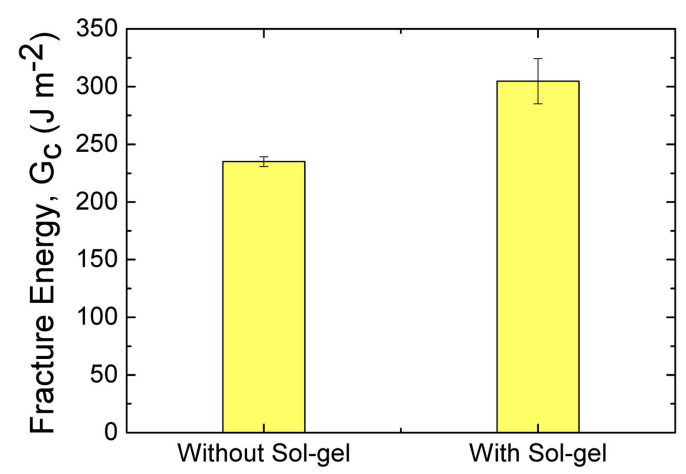

Fig. 5. Fracture energy of specimens with and without sol-gel derived hybrid layers after 1 minute of UV/Ozone treating time and 1 minute of UV/Ozone holding time.
The reason for increased fracture energy by UV/Ozone treatment was discussed above. By many previous studies, UV/Ozone treatment has been known to increase adhesive strength through removal of organic contaminants, increased surface wetting, surface activation, and introduction of variety of functional groups, such as ether group, carbonyl group, and carboxyl group. ${ }^{16-19)}$ All these factors contributed into making more hydrogen bonds available for the epoxy, resulting in better adhesion. For sol-gel derived hybrid layers, as Zhang et al. and many other researchers suggest, the reason for increased fracture energy is formation of chemical bonding at the interface. ${ }^{20,21)}$ Stable chemical bondings, such as shown in Fig. 3 (c) will increase the adhesion between PI and epoxy. For epoxy and silicon, GPTMS+TPOZ will create stable chemical bondings, therefore increasing the adhesion. Together with many beneficial effects of UV/ Ozone treatment, applying sol-gel derived hybrid layers will increase the fracture energy furthermore since removal of organic contaminants, surface characterization, and better surface wetting will help sol-gel derived hybrid layers to create more stable chemical networks.

\subsection{Humidity reliability enhancement results}

In order to determine the effect of UV/Ozone treatment and sol-gel derived hybrid layers for humidity reliability enhancement, subcritical test was performed. Subcritical test was first performed for only UV/Ozone treated specimens and its results are shown in Fig. 6. The red line describes the subcritical behavior of UV/Ozone untreated sample with its threshold applied strain energy release rate being $12 \mathrm{~J} \mathrm{~m}^{-2}$ while the blue line is for the UV/Ozone treated specimen with $14 \mathrm{~J} \mathrm{~m}^{-2}$ of threshold applied strain energy release rate. The fact that both threshold applied strain energy release rate is similar for UV/Ozone treated and untreated specimen means that UV/Ozone treatment alone cannot protect the specimen from subcritical crack growth due to humidity. This is a reasonable result since applying UV/Ozone treatment does not change the nature epoxy on the interfaces, but rather helps epoxy to form more hydrogen bonds to the substrate. This is not effective against moisture penetration since water breaks down hydrogen bonds easily. Also, water moves through a network of nanopores in epoxy that is inherent in its structure. The average size of nanopores diameter is about $5 \sim 6.1 \AA$, whereas the approximate diameter of water molecule is about $3 \AA^{22)}$ Hence, even with UV/Ozone treatment, water molecules will break down hydrogen bonds and move through a network of nanopores that is in epoxy structure.

Therefore, sol-gel derived hybrid layers were applied to 


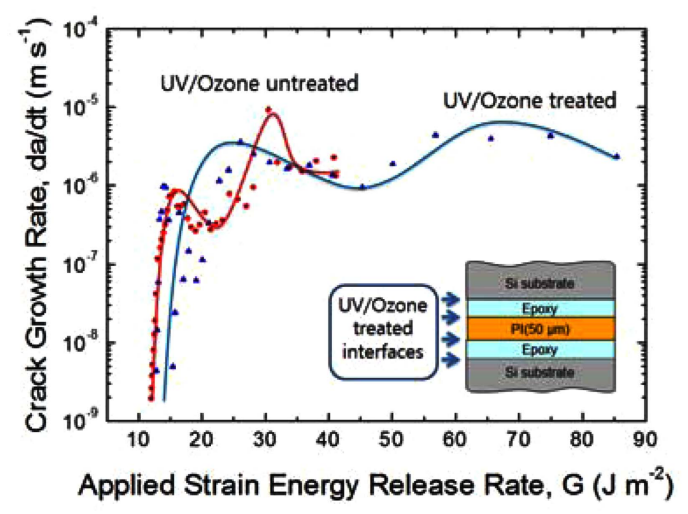

Fig. 6. Subcritical graph for UV/Ozone untreated and UV/Ozone treated specimens.

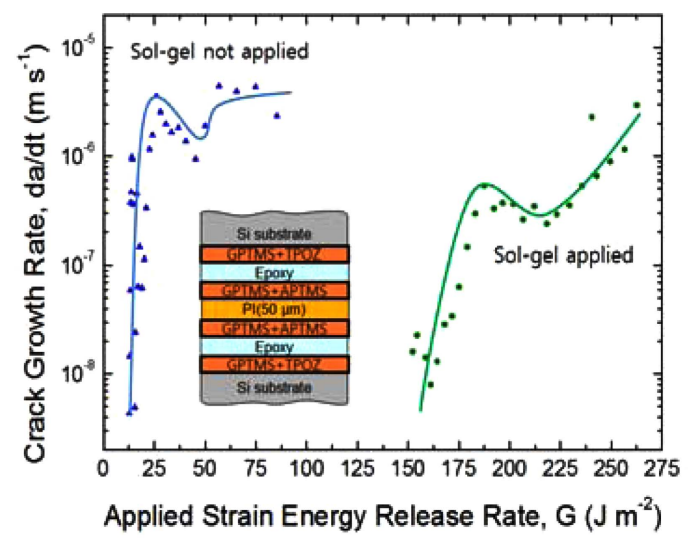

Fig. 7. Subcritical graph for sol-gel derived hybrid layer applied and sol-gel derived hybrid layer not applied specimens with 1 minute of UV/Ozone treating time and 1 minute of UV/ Ozone holding time.

enhance the humidity reliability of the specimens. For this experiment, UV/Ozone treatment was applied with 1 minute of treating time and 1 minute of holding time. The subcritical test result of this UV/Ozone treated and sol-gel derived hybrid layers applied specimens is shown in Fig. 7. The threshold applied strain energy release rate increased greatly, implying that the humidity reliability of the specimen improved drastically. The threshold applied strain energy release rate of sol-gel derived hybrid layers applied specimen increased to $152 \mathrm{~J} \mathrm{~m}^{-2}$ from $14 \mathrm{~J} \mathrm{~m}^{-2}$. Unlike UV/ Ozone treatment, applying sol-gel derived hybrid layers produce extra layers that create water-resistant bond at the interface. This bond will resist from breaking down due to moisture penetration more than hydrogen bonds between epoxy and substrates, therefore increasing the threshold applied strain energy release rate. This, in turn, means that humidity reliability is enhanced greatly by applying sol-gel derived hybrid layers rather than UV/Ozone treatment.

\section{Conclusion}

In this research, adhesion and humidity reliability enhancement were achieved through UV/Ozone treatment and sol-gel derived hybrid layers. When both methods were applied to the specimen with structures like Fig. 1 (a), the adhesion increased from $87 \mathrm{~J} \mathrm{~m}^{-2}$ to $304 \mathrm{~J} \mathrm{~m}^{-2}$ while the threshold applied strain energy release rate increased from 14 $\mathrm{J} \mathrm{m}^{-2}$ to $152 \mathrm{~J} \mathrm{~m}^{-2}$. These results indicate the effectiveness of applying UV/Ozone treatment and sol-gel derived hybrid layer. Another important findings from this research is the specific advantages of UV/Ozone treatment and sol-gel derived hybrid layers. Applying UV/Ozone treatment resulted in better enhancement in fracture energy but humidity reliability was not improved. However, applying sol-gel derived hybrid layers proved to be more effective for humidity reliability enhancement than UV/Ozone treatment while the adhesion increase was also found. Hence, the initial goal of this research was achieved by using UV/ Ozone treatment and sol-gel derived hybrid layers.

\section{Acknowledgement}

This research was supported by Basic Science Research Program through the National Research Foundation of Korea (NRF) funded by the Ministry of Education, Science and Technology (2012006072), and the MCP Core Technologies for the Next Generation Project of the Ministry of Knowledge Economy of Korea.

\section{References}

1. D. Hung, F. Liao, S. Molesa, D. Redinger and V. Subramanian, "Plastic-Compatible Low Resistance Printable Gold Nanoparticle Conductors for Flexible Electronics", Journal of The Electrochemical Society, 150(7) G412-G417 (2003).

2. S. Ju, A. Facchetti, Y. Xuan, J. Liu, F. Ishikawa, P. Ye, C. Zhou, T. J. Marks and D. B. Janes, "Fabrication of fully transparent nanowire transistors for transparent and flexible electronics", Nature nanotechnology, 2, 378 (2007).

3. Y. Sun and J. A. Rogers, "Inorganiic Semiconductors for Flexible Electronics", Advanced Materials, 19, 1897 (2007).

4. K. Yano, A. Usuki, A. Okada, T. Kurauchi and O. Kamigaito, "Synthesis and properties of polyimide-clay hybrid", Journal of Polymer Science Part A: Polymer Chemistry, 31, 2493 (1993).

5. S. Luo, J. Leisen and C. P. Wong, "Study of mobility of water and polymer chain in epoxy and its influence on adhesion", Journal of applied polymer science, 85, 1 (2002).

6. C. E. Park, B. J. Han and H. E. Bair, "Humidity effects on adhesion strength between solder ball and epoxy underfills", Polymer, 38, 3811 (1997).

7. M. Shirangi and B. Michel, "Mechanism of Moisture Diffu- 
sion, Hygroscopic Swelling, and Adhesion Degradation in Epoxy Molding Compounds", Moisture Sensitivity of Plaastic Packages of IC Devices, 29-69 (2010).

8. D. C. Hu and H. C. Chen, "Humidity effect on polyimide film adhesion”, Journal of materials science, 27, 5262 (1992).

9. S. Luo and C. P. Wong, "Influence of temperature and humidity on adhesion of underfills for flip chip packaging", Components and Packaging Technologies, IEEE Transactions on 28, 88 (2005).

10. S. Y. Kim, S. S. Jo, J. S. Kang and Y. H. Kim, "Adhesion Enhancement of Thin Film Metals on Polyimide Substrates by Bias Sputtering”, J. Microelectron. Packag. Soc., 12(3), 207 (2005).

11. S. Y. Kim, Y. H. Kim and J. S. Yoon, "광반응 폴리이미드위 에 $\mathrm{RF}$ bias sputtering 방식으로 증착된 $\mathrm{Cr}$ 의 접착력에 관한 연 구”, Proc. International Microelectronics and Packaging Society Conference, Seoul, 171, The Korean Microelectronics and Packaging Society (KMEPS) (2001). (in Korean)

12. N. S. McIntyre, R. D. Davidson, T. L. Walzak, R. Williston, M. Westcott and A. Pekarsky, "Uses of ultraviolet/ozone for hydrocarbon removal: Applications to surfaces of complex composition or geometry", Journal of Vacuum Science \& Technology A: Vacuum, Surfaces, and Films, 9, 1355 (1991).

13. M. S. Olilver, K. Y. Blohowiak and R. H. Dauskardt, "Molecular structure and fracture properties of $\mathrm{ZrO} \mathrm{X} /$ Epoxysilane hybrid films", Journal of sol-gel science and technology, 55, 360 (2010).

14. M. F. Kannine, "An augmented double cantilever beam model for studying crack propagation and arrest", International Journal of Fracture, 9(1), 83 (1973).
15. R. J. Hohlfelder, D. A. Maidenberg, R. H. Dauskardt, Y. Wei and W. Hutchinson, "Adhesion of benzocyclobutene-passivated silicon in epoxy layered structures", Journal of Materials Research, 16(1), 243 (2001).

16. J. R. Vig and J. W. LeBus, "UV/Ozone cleaning of surfaces", Parts, Hybrids, and Packaging, IEEE Transactions on 12, 365 (1976).

17. J. A. Poulis, J. C. Cool and E. H. P. Logtenberg, "UV/Ozone cleaning, a convenient alternative for high quality bonding preparation", International journal of adhesion and adhesives, 13, 89 (1993).

18. M. L. Sham, J. Li, P. C. Ma and J. K. Kim, "Cleaning and functionalization of polymer surfaces and nanoscale carbon fillers by uv/ozone treatment: A review", Journal of composite materials, 43, 1537 (2009).

19. D. O. H. Teare, C. Ton-That and R. H. Bradley, "Surface characterization and ageing of ultraviolet-ozone-treated polymers using atomic force microscopy and x-ray photoelectron spectroscopy", Surface and interface analysis, 29, 276 (2000).

20. S. Zhang, Z. Xianting, W. Yongsheng, C. Kui and W. Wenjian, "Adhesion strength of sol-gel derived fluoridated hydroxyapatite coatings", Surface and Coatings Technology, 200, 6350 (2006).

21. J. Liu, M. K. Chaudhury, D. H. Berry, J. E. Seebergh, J. H. Osborne, K. Y. Blohowiak, "Effect of surface morphology on crack growth at a sol-gel reinforced epoxy/aluminum interface", The Journal of Adhesion, 82, 487 (2006).

22. C. L. Soles and A. F. Yee, "A discussion of the molecular mechanisms of moisture transport in epoxy resins", Journal of Polymer Science Part B: Polymer Physics, 38, 792 (2000). 